MALAYA JOURNAL OF MATEMATIK

Malaya J. Mat. 09(02)(2021), 46-54.

http://doi.org/10.26637/mjm0902/006

\title{
Existence results for $\psi$-Caputo hybrid fractional integro-differential equations
}

\author{
NAAS AdJimi ${ }^{1}$, MAAMAR BENBAChIR ${ }^{* 2}$ AND KADDOUR GUERBATI ${ }^{3}$ \\ ${ }^{1,3}$ Laboratory of Mathematics and Applied Sciences. University of Ghardaia, 47000, Algeria \\ ${ }^{2}$ Faculty of Sciences, Saad Dahlab University, Blida 1, Algeria.
}

Received 12 November 2020; Accepted 17 March 2021

Abstract. In this paper, we study the existence of solutions for hybrid fractional integro-differential equations involving $\psi$ Caputo derivative. We use an hybrid fixed point theorem for a sum of three operators due to Dhage for proving the main results. An example is provided to illustrate main results.

AMS Subject Classifications: 34A08, 26A33, 34A34.

Keywords: Fractional order differential equations, multi-term time fractional derivative, fractional impulsive conditions, fractional order integral boundary conditions.

\section{Contents}

1 Introduction $\quad 4 \mathbf{4 6}$

$\begin{array}{llr}2 & \text { Background material } & \mathbf{4 7}\end{array}$

3 Main Results $\quad 48$

4 Application $\quad 5$

\section{Introduction}

Fractional differential equations are a generalization of the classical ordinary differential equations, they play a very important role in modeling of various fields of science and engineering, chemistry, physics, economics, biology, control, etc..., see [2, 3, 11, 12,17] and the references cited therein. The existence and uniqueness solution of fractional differential equations are studies by many authors, with different approaches, such as Riemann-Liouville, Caputo, Hadamard, Caputo-Hadamard, with various boundary conditions as nonlocal, integral, multipoint and hybrid, see for example [1,6]. In recent years, many researchers focused on developing the theoretical aspects and methods of solution of the hybrid fractional differential equations by using different kinds of fixed point, we refer the reader to the works $[5,9,16]$.

In [8] Almeida presented a new fractional differentiation operator named $\psi$-Caputo fractional operator. This type of differentiation depends on a kernel, and for particular choices of $\psi$, we obtain some well known fractional derivatives like Caputo, Caputo-Hadamard or Caputo, Erdélyi-Kober fractional derivatives. One can find some recent works on $\psi$-Caputo derivative in the following published papers and the references cited therein $[5,8,10,18]$.

*Corresponding author. Email address: naasadjimi@gmail.com (Naas Adjimi), mbenbachir2001@gmail.com (Maamar Benbachir), series guerbati_k@yahoo.com (Kaddour Guerbati) 
Existence results for $\psi$-Caputo hybrid fractional integro-differential equations

However, this domain reported on the existence solution for hybrid differential equations with $\psi$-Caputo fractional derivative still new.

Motivated by this fact, in this paper, we study the existence of solutions for $\psi$-Caputo hybrid fractional integro-differential equations of the form

$$
\left\{\begin{array}{l}
{ }^{c} \mathbb{D}_{a^{+}}^{\nu ; \psi}\left[\frac{z(\tau)-\sum_{k=1}^{m} \mathbb{I}_{a+}^{\sigma_{k} ; \psi} \mathbb{F}_{k}(\tau, z(\tau))}{\mathbb{G}(\tau, z(\tau))}\right]=\mathbb{H}(\tau, z(\tau)), \tau \in \mathrm{J}:=[a, b], \\
z(a)=0
\end{array}\right.
$$

where ${ }^{c} \mathbb{D}_{a^{+}}^{\nu ; \psi}$ is the $\psi$-Caputo fractional derivative of order $\nu \in(0,1], \mathbb{I}_{a^{+}}^{\theta ; \psi}$ is the $\psi$-Riemann-Liouville fractional integral of order $\theta>0, \theta \in\left\{\sigma_{1}, \sigma_{2}, \ldots, \sigma_{m}\right\}, \sigma_{k}>0, k=1,2, \ldots, m . \mathbb{G} \in C(\mathrm{~J} \times \mathbb{R}, \mathbb{R} \backslash\{0\})$ and $\mathbb{F}_{k}, \mathbb{H} \in$ $C(\mathrm{~J} \times \mathbb{R}, \mathbb{R}),(k=1,2, \ldots, m)$.

The paper is organized as follows. In section 2, we present some definitions of fractional calculus and lemmas. In section 3, we prove the existence of solutions for problem (1.1) via hybrid fixed point theorems in Banach algebra due to Dhage. In section 4, an example is provided to check the applicability of the theoretical findings.

\section{Background material}

First, we introduce the essential functional spaces that we will adopt in this paper. We denote by $\mathfrak{C}([a, b], \mathbb{R})$ the Banach space of all continuous functions $z$ from $[a, b]$ into $\mathbb{R}$ with the supremum norm

$$
\|z\|_{\mathfrak{C}}=\sup _{\tau \in[a, b]}|z(\tau)|
$$

and the multiplication in $\mathfrak{C}$ by

$$
(z y)(\tau)=z(\tau) y(\tau)
$$

Clearly, $\mathfrak{C}$ is a Banach algebra with respect to the supremum norm and multiplication in it. Now, we present some facts from the theory of fractional calculus.

Definition 2.1 ([7, 12]). For $\nu>0$, the left-sided $\psi$-Riemann-Liouville fractional integral of order $\nu$ for an integrable function $z:[a, b] \longrightarrow \mathbb{R}$ with respect to another function $\psi:[a, b] \longrightarrow \mathbb{R}$ that is an increasing differentiable function such that $\psi^{\prime}(\tau) \neq 0$, for all $\tau \in \mathrm{J}$ is defined as follows

$$
\mathbb{I}_{a^{+}}^{\nu ; \psi} z(\tau)=\frac{1}{\Gamma(\nu)} \int_{a}^{\tau} \psi^{\prime}(s)(\psi(\tau)-\psi(s))^{\nu-1} z(s) \mathrm{ds}
$$

where $\Gamma(\cdot)$ is the (Euler's) Gamma function defined by

$$
\Gamma(\nu)=\int_{0}^{+\infty} \tau^{\nu-1} e^{-\tau} \mathrm{d} \tau, \quad \nu>0 .
$$

Definition 2.2 ([7]). Let $n \in \mathbb{N}$ and let $\psi, z \in \mathfrak{C}^{n}([a, b], \mathbb{R})$ be two functions such that $\psi$ is increasing and $\psi^{\prime}(\tau) \neq 0$, for all $\tau \in \mathrm{J}$. The left-sided $\psi$-Riemann-Liouville fractional derivative of a function $z$ of order $\nu$ is defined by

$$
\begin{aligned}
\mathbb{D}_{a^{+}}^{\nu ; \psi} z(\tau) & =\left(\frac{1}{\psi^{\prime}(\tau)} \frac{d}{d t}\right)^{n} \mathbb{I}_{a^{+}}^{n-\nu ; \psi} z(\tau) \\
& =\frac{1}{\Gamma(n-\nu)}\left(\frac{1}{\psi^{\prime}(\tau)} \frac{d}{d t}\right)^{n} \int_{a}^{\tau} \Xi(s) \mathrm{ds}
\end{aligned}
$$

where $n=[\nu]+1$ and $\Xi(s)=\psi^{\prime}(s)(\psi(\tau)-\psi(s))^{n-\nu-1} z(s)$. 
Naas Adjimi, Maamar Benbachir and Kaddour Guerbati

Definition 2.3 ([7]). Let $n \in \mathbb{N}$ and let $\psi, z \in \mathfrak{C}^{n}([a, b], \mathbb{R})$ be two functions such that $\psi$ is increasing and $\psi^{\prime}(\tau) \neq 0$, for all $\tau \in \mathrm{J}$. The left-sided $\psi$-Caputo fractional derivative of $z$ of order $\nu$ is defined by

$$
{ }^{c} \mathbb{D}_{a^{+}}^{\nu ; \psi} z(\tau)=\mathbb{I}_{a^{+}}^{n-\nu ; \psi}\left(\frac{1}{\psi^{\prime}(\tau)} \frac{d}{d t}\right)^{n} z(\tau),
$$

where $n=[\nu]+1$ for $\nu \notin \mathbb{N}, n=\nu$ for $\nu \in \mathbb{N}$.

Lemma 2.4 ([7]). Let $\nu, \beta>0$, and $z \in \mathfrak{C}([a, b], \mathbb{R})$. Then for each $\tau \in \mathrm{J}$ we have

(1) ${ }^{c} \mathbb{D}_{a^{+}}^{\nu ; \psi} \mathbb{I}_{a^{+}}^{\nu ; \psi} z(\tau)=z(\tau)$,

(2) $\mathbb{I}_{a^{+}}^{\nu ; \psi} \mathbb{D}_{a^{+}}^{\nu ; \psi} z(\tau)=z(\tau)-z(a), \quad 0<\nu \leq 1$,

(3) $\mathbb{I}_{a^{+}}^{\nu ; \psi}(\psi(\tau)-\psi(a))^{\beta-1}=\frac{\Gamma(\beta)}{\Gamma(\beta+\nu)}(\psi(\tau)-\psi(a))^{\beta+\nu-1}$,

(4) ${ }^{c} \mathbb{D}_{a^{+}}^{\nu ; \psi}(\psi(\tau)-\psi(a))^{\beta-1}=\frac{\Gamma(\beta)}{\Gamma(\beta-\nu)}(\psi(\tau)-\psi(a))^{\beta-\nu-1}$,

$(5)^{c} \mathbb{D}_{a^{+}}^{\nu ; \psi}(\psi(\tau)-\psi(a))^{k}=0$, for all $k \in\{0, \ldots, n-1\}, n \in \mathbb{N}$.

Theorem 2.5. Let $S$ be a closed convex, bounded and nonempty subset of a Banach algebra $\mathcal{X}$, and let $\mathcal{A}, \mathcal{C}$ : $\mathcal{X} \longrightarrow \mathcal{X}$ and $\mathcal{B}: S \longrightarrow \mathcal{X}$ be three operators such that

(a) $\mathcal{A}$ and $\mathcal{C}$ are Lipschitzian with Lipschitz constants $\delta$ and $\xi$, respectively,

(b) $\mathcal{B}$ is compact and continuous,

(c) $x=\mathcal{A} x \mathcal{B} y+\mathcal{C} x \Rightarrow x \in S$ for all $y \in S$,

(d) $\delta M+\xi<1$ where $M=\|\mathcal{B}(S)\|$.

Then the operator equation $\mathcal{A} x \mathcal{B} x+\mathcal{C} x=x$ has a solution in $S$.

\section{Main Results}

Before proceeding to the main results, we start by the following lemma.

Lemma 3.1. Let $\nu \in(0,1]$ be fixed and functions $\mathbb{F}_{i},(i=1, \cdots, n), \mathbb{G}, \mathbb{H}$ satisfy problem (1.1). Then the function $z \in \mathfrak{C}([a, b], \mathbb{R})$ is a solution of the hybrid fractional integro-differential problem (1.1) if and only if it satisfies the integral equation

$$
\begin{aligned}
z(\tau)= & \mathbb{G}(\tau, z(\tau))\left[\mathbb{M}_{\psi}+\mathbb{I}_{a^{+}}^{\nu ; \psi} \mathbb{H}(\tau, z(\tau))\right] \\
& +\sum_{k=1}^{m} \mathbb{I}_{a^{+}}^{\sigma_{k} ; \psi} \mathbb{F}_{k}(\tau, z(\tau)), \quad \tau \in[a, b],
\end{aligned}
$$

where

$$
\mathbb{M}_{\psi}=\frac{-\sum_{k=1}^{m} \mathbb{I}_{a^{+}}^{\sigma_{k} ; \psi} \mathbb{F}_{k}(a, 0)}{\mathbb{G}(a, 0)}
$$

For the proof of Lemma 3.1, it is useful to refer to [13, 14].

Theorem 3.2. Assume that:

$\left(H_{1}\right)$ Let the functions $\mathbb{G}: \mathrm{J} \times \mathbb{R} \rightarrow \mathbb{R} \backslash\{0\}$ and, $\mathbb{F}_{k}, \mathbb{H}: \mathrm{J} \times \mathbb{R} \rightarrow \mathbb{R}, k=0,1,2, \ldots, m$ are continuous

$\left(H_{2}\right)$ There exists two positive functions $\mathbb{L}_{\mathbb{F}_{k}}, \mathbb{L}_{\mathbb{G}}, \quad k=0,1, \ldots, m$ with bounds $\left\|\mathbb{L}_{\mathbb{F}_{k}}\right\|$ and $\left\|\mathbb{L}_{\mathbb{G}}\right\|$, $k=0,1,2, \ldots, m$, respectively, such that 
Existence results for $\psi$-Caputo hybrid fractional integro-differential equations

$$
\begin{array}{r}
\left|\mathbb{F}_{k}(\tau, z(\tau))-\mathbb{F}_{k}(\tau, \bar{z}(\tau))\right| \leq \mathbb{L}_{\mathbb{F}_{k}(\tau)|z-\bar{z}|} \\
k=0,1 \ldots, m
\end{array}
$$

and

$$
|\mathbb{G}(\tau, z(\tau))-\mathbb{G}(\tau, \bar{z}(\tau))| \leq \mathbb{L}_{\mathbb{G}}(\tau)|z-\bar{z}|,
$$

for all $(\tau, z, \bar{z}) \in \mathrm{J} \times \mathbb{R} \times \mathbb{R}$.

$\left(H_{3}\right)$ There exist a function $p \in \mathfrak{C}\left(J, \mathbb{R}_{+}\right)$and a continuous nondecreasing function $\Omega:[0, \infty) \rightarrow(0, \infty)$ such that

$$
|\mathbb{H}(\tau, z(\tau))| \leq p(\tau) \Omega(|z|)
$$

for all $\tau \in \mathrm{J}$ and $z \in \mathbb{R}$.

$\left(H_{4}\right)$ There exists a number $r>0$ such that

$$
r \geq \frac{\mathbb{G}^{*} \Lambda+\ell_{\psi}^{\sigma_{k}} \mathbb{F}_{k}^{*}}{1-\left\|\mathbb{L}_{\mathbb{G}}\right\| \Lambda-\ell_{\psi}^{\sigma_{k}}\left\|\mathbb{L}_{\mathbb{F}_{k}}\right\|}
$$

and

$$
\left\|\mathbb{L}_{\mathbb{G}}\right\| \Lambda+l_{\psi}^{\sigma_{k}} \mathbb{F}_{k}^{*}<1
$$

where $\mathbb{F}_{k}^{*}=\sup _{\tau \in \mathrm{J}}\left|\mathbb{F}_{k}(\tau, 0)\right|$, and $\mathbb{G}^{*}=\sup _{\tau \in \mathrm{J}}|\mathbb{G}(\tau, 0)|, k=0,1,2, \ldots, m$, and

$$
\Lambda=\left|\mathbb{M}_{\psi}\right|+\Omega(r)\|p\| l_{\psi}^{\nu},
$$

Then hybrid fractional integro-differential problem (1.1) has a least one solution defined on J.

Proof. In order to use Dhage's fixed-point theorem to prove our main result, we define a subset $\mathbb{S}_{r}$ of $\mathfrak{C}$ by

$$
\mathbb{S}_{r}=\left\{z \in \mathfrak{C}:\|z\|_{\mathfrak{C}} \leq r\right\},
$$

with $r$ is a constant defined by hypothesis $H_{4}$.

Notice that $\mathbb{S}_{r}$ is closed, convex and bounded subset of $\mathfrak{C}$. Define three operators $\mathbb{A}, \mathbb{C}: \mathfrak{C} \longrightarrow \mathfrak{C}$ and $\mathbb{B}: \mathbb{S}_{r} \longrightarrow \mathfrak{C}$ by

$$
\left\{\begin{array}{l}
\mathbb{A} z(\tau)=\mathbb{G}(\tau, z(\tau)), \\
\mathbb{B} z(\tau)=\mathbb{M}_{\psi}+\mathbb{I}_{a^{+}}^{\nu ;} \mathbb{H}(\tau, z(\tau)),
\end{array} \quad \tau \in \mathrm{J},\right.
$$

and

$$
\mathbb{C} z(\tau)=\sum_{k=1}^{m} \mathbb{I}_{a^{+}}^{\sigma_{k} ; \psi} \mathbb{F}_{k}(\tau, z(\tau)), \quad \tau \in \mathrm{J} .
$$

Then (3.1) in operator form becomes

$$
z(\tau)=\mathbb{A} z(\tau) \mathbb{B} z(\tau)+\mathbb{C} z(\tau), \quad \tau \in \mathrm{J} .
$$




\section{Naas Adjimi, Maamar Benbachir and Kaddour Guerbati}

We shall prove that the operators $\mathbb{A}, \mathbb{B}$ and $\mathbb{C}$ satisfy the conditions of Theorem 2.5 . For the sake of clarity, we split the proof into a sequence of steps.

Step 1: First, we show that $\mathbb{A}$ and $\mathbb{C}$ are Lipschitzian on $\mathfrak{C}$. Let $z, \bar{z} \in \mathfrak{C}$. then by (H2), for $\tau \in[a, b]$, we have

$$
\begin{aligned}
|\mathbb{A} z(\tau)-\mathbb{A} \bar{z}(\tau)| & =|\mathbb{G}(\tau, z(\tau))-\mathbb{G}(\tau, \bar{z}(\tau))| \\
& \leq \mathbb{L}_{\mathbb{G}}(\tau)\|z(\tau)-\bar{z}(\tau)\|_{\mathfrak{C}} .
\end{aligned}
$$

Taking supremum over $\tau \in[a, b]$, we obtain

$$
\|\mathbb{A} z-\mathbb{A} \bar{z}\|_{\mathfrak{C}} \leq\left\|\mathbb{L}_{\mathbb{G}}\right\|\|z(\tau)-\bar{z}(\tau)\|_{\mathfrak{C}}
$$

for all $z, \bar{z} \in \mathfrak{C}$. Therefore, $\mathbb{A}$ is a Lipschitzian on $\mathfrak{C}$ with Lipschitz constant $\mathbb{L}_{\mathbb{G}}$. Also, for any $z, \bar{z} \in \mathfrak{C}$., we have

$$
\begin{aligned}
|\mathbb{C} z(\tau)-\mathbb{C} \bar{z}(\tau)| & \leq \sum_{k=1}^{m} \mathbb{I}_{a^{+}}^{\sigma_{k} ; \psi}\left|\mathbb{F}_{k}(\tau, z(\tau))-\mathbb{F}_{k}(\tau, \bar{z}(\tau))\right| \\
& \leq \sum_{k=1}^{m} \mathbb{I}_{a^{+}}^{\sigma_{k} ; \psi} \mathbb{L}_{\mathbb{F}_{k}}(\tau)\|z(\tau)-\bar{z}(\tau)\|_{\mathfrak{C}} \\
& \leq \sum_{k=1}^{m} \frac{(\psi(b)-\psi(a))^{\sigma_{k}}}{\Gamma\left(\sigma_{k}+1\right)}\left\|\mathbb{L}_{\mathbb{F}_{k}}\right\|\|z(\tau)-\bar{z}(\tau)\|_{\mathfrak{C}} .
\end{aligned}
$$

Hence, we have

$$
\|\mathbb{C} z-\mathbb{C} \bar{z}\|_{\mathfrak{C}} \leq \ell_{\psi}^{\sigma_{k}}\left\|\mathbb{L}_{\mathbb{F}_{k}}\right\|\|z(\tau)-\bar{z}(\tau)\|_{\mathfrak{C}}
$$

Which means that $\mathbb{C}$ is a Lipschitzian on $\mathfrak{C}$ with Lipschitz constant $\ell_{\psi}^{\sigma_{k}}\left\|\mathbb{L}_{\mathbb{F}_{k}}\right\|$.

Step 2: We show that $\mathbb{B}$ is completely continuous on $\mathbb{S}_{r}$. The continuity of $\mathbb{B}$ follows by the continuity of $\mathbb{H}$. Now, it is sufficient to show that $\mathbb{B}$ is uniformly bounded and equicontinuous on $\mathbb{S}_{r}$. On the other hand, Keeping in mind the definition of the operator $\mathbb{B}$ on $[a, b]$ together with assumption $(\mathrm{H} 3)$. For any $z \in \mathbb{S}_{r}$ we can get

$$
\begin{aligned}
|\mathbb{B} z(\tau)| & \leq\left|\mathbb{M}_{\psi}\right|+\int_{a}^{\tau} \frac{\psi^{\prime}(s)(\psi(\tau)-\psi(s))^{\nu-1}}{\Gamma(\nu)} \mid \mathbb{H}(s, z(s) \mid \mathrm{ds} \\
& \leq\left|\mathbb{M}_{\psi}\right| \int_{a}^{\tau} \frac{\psi^{\prime}(s)(\psi(\tau)-\psi(s))^{\nu-1}}{\Gamma(\nu)} \Omega(r) p(s) \mathrm{ds} \\
& \leq\left|\mathbb{M}_{\psi}\right|+\Omega(r)\|p\| \int_{a}^{\tau} \frac{\psi^{\prime}(s)(\psi(\tau)-\psi(s))^{\nu-1}}{\Gamma(\nu)} \mathrm{ds} \\
& \leq\left|\mathbb{M}_{\psi}\right|+\frac{(\psi(b)-\psi(a))^{\nu}}{\Gamma(\nu+1)} \Omega(r)\|p\| \\
& =\left|\mathbb{M}_{\psi}\right|+\Omega(r)\|p\| \ell_{\psi}^{\nu} .
\end{aligned}
$$

Hence

$$
\|\mathbb{B} z\|_{\mathfrak{C}} \leq\left|\mathbb{M}_{\psi}\right|+\Omega(r)\|p\| \ell_{\psi}^{\nu} .
$$

Thus $\|\mathbb{B} z\| \leq \Lambda$ with $\Lambda$ given in (3.8), for all $z \in \mathbb{S}_{r}$. This shows that $\mathbb{B}$ is uniformly bounded on $\mathbb{S}_{r}$.

Now, we will show that $\mathbb{B}\left(\mathbb{S}_{r}\right)$ is an equicontinuous set in $\mathfrak{C}$.

Let $\tau_{1}, \tau_{2} \in \mathrm{J}$ with $\tau_{1}<\tau_{2}$. Then for any $z \in \mathbb{S}_{r}$, by (3.5) we get

$$
\begin{aligned}
\left|\mathbb{B} z\left(\tau_{2}\right)-\mathbb{B} z\left(\tau_{1}\right)\right| & \leq\left|\int_{a}^{\tau_{2}} \Theta_{\tau_{2}}(s) \mathrm{ds}-\int_{a}^{\tau_{1}} \Theta_{\tau_{1}}(s) \mathrm{ds}\right| \\
& \leq \frac{\Omega(r)\|p\|}{\Gamma(\nu)} \int_{a}^{\tau_{1}} \Delta_{1}(s) \mathrm{ds}+\frac{\Omega(r)\|p\|}{\Gamma(\nu)} \int_{\tau_{1}}^{\tau_{2}} \Delta_{2}(s) \mathrm{ds},
\end{aligned}
$$


Existence results for $\psi$-Caputo hybrid fractional integro-differential equations

where $\Theta_{\tau_{2}}(s)=\frac{\psi(s)\left(\psi\left(\tau_{2}\right)-\psi(s)\right)^{\nu-1}}{\Gamma(\nu)} \mathbb{H}(\tau, z(\tau)), \Theta_{\tau_{1}}(s)=\frac{\psi(s)\left(\psi\left(\tau_{1}\right)-\psi(s)\right)^{\nu-1}}{\Gamma(\nu)} \mathbb{H}(\tau, z(\tau))$, $\Delta_{1}(s)=\psi^{\prime}(s)\left[\left(\psi\left(\tau_{1}\right)-\psi(s)\right)^{\nu-1}-\left(\psi\left(\tau_{2}\right)-\psi(s)\right)^{\nu-1}\right]$ and $\Delta_{2}(s)=\psi^{\prime}(s)\left(\psi\left(\tau_{2}\right)-\psi(s)\right)^{\nu-1}$.

It is clear that the right-hand side of (3.9) is independent of $z$. Therefore, as $\tau_{2} \rightarrow \tau_{1}$, inéquality (3.9) tends zeros. As consequence of the Arzela-Ascoli theorem, $\mathbb{B}$ is a completely continuous operator on $\mathbb{S}_{r}$.

Step 3: The hypothesis (c) of Theorem 2.5 is satisfied.

Let $z \in \mathfrak{C}$ and $y \in \mathbb{S}_{r}$ be arbitrary elements such that $z=\mathbb{A} z \mathbb{B} y+\mathbb{C} z$. Then we have

$$
\begin{aligned}
|z(\tau)| & \leq|\mathbb{A} z(\tau)||\mathbb{B} y(\tau)|+|\mathbb{C} z(\tau)| \\
& \leq|\mathbb{G}(\tau, z(\tau))|\left\{\mathbb{M}_{\psi}+\mathbb{I}_{a^{+}}^{\nu ; \psi}|\mathbb{H}(\tau, y(\tau))|\right\}+\sum_{k=1}^{m} \mathbb{I}_{a^{+}}^{\sigma_{k} ; \psi}\left|\mathbb{F}_{k}(\tau, z(\tau))\right| \\
& \leq(|\mathbb{G}(\tau, z(\tau))-\mathbb{G}(\tau, 0)| \\
& +|\mathbb{G}(\tau, 0)|)\left\{\mathbb{M}_{\psi}+\mathbb{I}_{a^{+}}^{\nu ; \psi}|\mathbb{H}(\tau, y(\tau))|\right\}+\sum_{k=1}^{m} \mathbb{I}_{a^{+}}^{\sigma_{k} ; \psi} \mid\left(\left|\mathbb{F}_{k}(\tau, z(\tau))-\mathbb{F}_{k}(\tau, 0)\right|+\left|\mathbb{F}_{k}(\tau, 0)\right|\right) \\
& \leq\left(\left\|\mathbb{L}_{\mathbb{G}}\right\|\|z\|_{\mathfrak{C}}+\mathbb{G}^{*}\right)\left[\left|\mathbb{M}_{\psi}\right|+\Omega(r)\|p\| \ell_{\psi}^{\nu}\right]+\ell_{\psi}^{\sigma_{k}}\left(\left\|\mathbb{L}_{\mathbb{F}_{k}}\right\|\|z\|_{\mathfrak{C}}+\mathbb{F}_{k}^{*}\right) .
\end{aligned}
$$

Thus,

$$
|z(\tau)| \leq\left(\left\|\mathbb{L}_{\mathbb{G}}\right\|\|z\|_{\mathfrak{C}}+\mathbb{G}^{*}\right) \Lambda+\ell_{\psi}^{\sigma_{k}}\left(\left\|\mathbb{L}_{\mathbb{F}_{k}}\right\|\|z\|_{\mathfrak{C}}+\mathbb{F}_{k}^{*}\right)
$$

Taking the supremum over $\tau$,

$$
\|z\| \leq \frac{\mathbb{G}^{*} \Lambda+\ell_{\psi}^{\sigma_{k}} \mathbb{F}_{k}^{*}}{1-\left\|\mathbb{L}_{\mathbb{G}}\right\| \Lambda-\ell_{\psi}^{\sigma_{k}}\left\|\mathbb{L}_{\mathbb{F}_{k}}\right\|} \leq r .
$$

Step 4: Finally we show that $\delta M+\xi<1$, that is, (d) of Theorem 2.5 holds.

Since

$$
M=\|B(S)\|=\sup _{z \in S}\left\{\sup _{\tau \in J}|B z(t)|\right\} \leq \Lambda
$$

and so

$$
\left\|\mathbb{L}_{\mathbb{G}}\right\| M+\ell_{\psi}^{\sigma_{k}}\left\|\mathbb{L}_{\mathbb{F}_{k}}\right\| \leq\left\|\mathbb{L}_{\mathbb{G}}\right\| \Lambda+\ell_{\psi}^{\sigma_{k}}\left\|\mathbb{L}_{\mathbb{F}_{k}}\right\|<1
$$

with $\delta=\left\|\mathbb{L}_{\mathbb{G}}\right\|, \xi=\ell_{\psi}^{\sigma_{k}}\left\|\mathbb{L}_{\mathbb{F}_{k}}\right\|$. Thus all the conditions of Theorem 2.5 are satisfied and hence the operator equation $z=\mathcal{A} z \mathcal{B} z+\mathcal{C} z$ has a solution in $\mathbb{S}_{r}$. As a result, problem (1.1) has a solution on J.

\section{Application}

In this section, we present an example to show the applicability of the main result.

Example 4.1. Consider the following hybrid fractional integro-differential equation:

$$
\left\{\begin{array}{l}
{ }^{c} \mathbb{D}_{a^{+}}^{\frac{1}{2} ; \psi}\left[\frac{z(\tau)-\sum_{k=1}^{m} \mathbb{I}_{a+}^{\sigma} ; \psi}{\mathbb{G}(\tau, z(\tau))} \mathbb{F}_{k}(\tau, z(\tau))\right. \\
\tau \in \mathrm{J}:=[0,1] \\
z(a)=0 .
\end{array}\right.
$$

We take

$$
\nu=\frac{1}{2}, \quad m=3, \quad \sigma_{1}=\frac{1}{2}, \quad \sigma_{2}=\frac{3}{2}, \quad \sigma_{3}=\frac{5}{2}
$$


Naas Adjimi, Maamar Benbachir and Kaddour Guerbati

$$
\begin{aligned}
\sum_{k=1}^{3} \mathbb{I}_{a^{+}}^{\sigma_{k} ; \psi} \mathbb{F}_{k}(\tau, z(\tau))= & \mathbb{I}_{a^{+}}^{\frac{1}{2} ; \psi} \frac{\tau}{10}\left(z(\tau)+e^{-\tau}\right) \\
& \quad+\mathbb{I}_{a^{+}}^{\frac{3}{2} ; \psi} \frac{\tau \cos \tau}{12\left(1+e^{\tau}\right)}\left(\frac{|z(\tau)|}{1+|z(\tau)|}+\frac{\tau}{\tau+1}\right) \\
& \quad+\mathbb{I}_{a^{+}}^{\frac{5}{2} ; \psi} \frac{3 \sin \pi \tau}{4+\tau}\left(\frac{|z(\tau)|}{5+|z(\tau)|}+\cos \tau\right) \\
\psi(\tau, z(\tau))= & \frac{\tau}{2}(\tau+1), \tau \in[0,1] \\
\mathbb{G}(\tau, z(\tau))= & \frac{6 \sqrt{\pi} \sin ^{2}(\pi \tau)}{(\tau+5)} \frac{z(\tau)}{1+z(\tau)}+\frac{1}{2}, \\
\mathbb{H}(\tau, z(\tau)))= & \frac{1}{\sqrt{36+t^{2}}}\left(\frac{|z|}{(4|z|+1)}+\frac{z^{2}}{|z|+1}+\frac{1}{4}\right) .
\end{aligned}
$$

We can show that

$$
\begin{aligned}
\left|\mathbb{F}_{1}(\tau, z(\tau))-\mathbb{F}_{1}(\tau, \bar{z}(\tau))\right| & \leq \frac{\tau}{10}|z-\bar{z}|, \\
\left|\mathbb{F}_{2}(\tau, z(\tau))-\mathbb{F}_{2}(\tau, \bar{z}(\tau))\right| & \leq \frac{\tau}{12\left(1+e^{\tau}\right)}|z-\bar{z}|, \\
\left|\mathbb{F}_{3}(\tau, z(\tau))-\mathbb{F}_{3}(\tau, \bar{z}(\tau))\right| & \leq \frac{3}{20+5 \tau}|z-\bar{z}|, \\
|\mathbb{G}(\tau, z(\tau)) \mathbb{G}(\tau, \bar{z}(\tau))-| & \leq \frac{6 \sqrt{\pi}}{(\tau+5)}|z-\bar{z}|, \\
\mid \mathbb{H}(\tau, z(\tau)))-\mathbb{H}(\tau, \bar{z}(\tau))) \mid & =\frac{1}{\sqrt{36+t^{2}}}\left(|z|+\frac{1}{2}\right),
\end{aligned}
$$

where

Hence we have

$$
\Omega(|z|)=|z|+1, \quad p(\tau)=\frac{1}{\sqrt{36+t^{2}}}
$$

$$
\mathbb{L}_{\mathbb{G}}(\tau)=\frac{6 \sqrt{\pi}}{(\tau+5)}, \quad \mathbb{F}_{1}=\frac{\tau}{10}, \quad \mathbb{F}_{2}=\frac{\tau}{12\left(1+e^{\tau}\right)}, \quad \mathbb{F}_{3}=\frac{3}{20+\tau}
$$

Then

$$
\begin{array}{r}
\left\|\mathbb{L}_{\mathbb{G}}\right\|=\frac{6 \sqrt{\pi}}{5}, \quad\left\|\mathbb{L}_{\mathbb{F}_{1}}\right\|=\frac{1}{10}, \quad\left\|\mathbb{L}_{\mathbb{F}_{2}}\right\|=\frac{1}{12(1+e)}, \\
\left\|\mathbb{L}_{\mathbb{F}_{3}}\right\|=\frac{3}{20}, \quad\|p\|=\frac{1}{6}, \quad l_{\psi}^{\nu}=\frac{2}{\sqrt{\pi}}, \\
l_{\psi}^{\sigma_{k}}\left\|\mathbb{L}_{\mathbb{F}_{k}}\right\|=\frac{81(1+e)+25}{450 \sqrt{\pi}(1+e)}, \quad l_{\psi}^{\sigma_{k}} \mathbb{F}_{k}^{*}=\frac{58}{75 \sqrt{\pi} e^{2}} \\
\mathbb{M}_{\psi}=\frac{234(1+e)+100}{225 \sqrt{\pi}(1+e)},
\end{array}
$$

and

$$
\mathbb{F}_{k}^{*}=\sup _{z \in J}\left|\mathbb{F}_{k}(\tau, 0)\right|=\frac{1}{5 e^{2}}, \quad \mathbb{G}^{*}=\sup _{z \in J}|\mathbb{G}(\tau, 0)|=\frac{1}{2}, \quad k=1,2,3 .
$$

By using Matlab program, it follows by (3.6) and (3.7) that the constant $r$ satisfies the inequality $0.7411<r<$ 0.9970. As all the assumptions of Theorem (3.2) are satisfied then the problem (4.1) has at least one solution on J. 
Existence results for $\psi$-Caputo hybrid fractional integro-differential equations

\section{References}

[1] M.I. AвbAS, Existence results for Hadamard and Riemann-Liouville functional fractional neutral integrodifferential equations and finite delay, Filomat, 32(13) (2018), 4611-4618.

[2] S. Abbas, M. Benchohra And G. M. N'GuéréKata, Topics in Fractional Differential Equations, Developments in Mathematics, 27, Springer, New York, 2012.

[3] S. Abbas, M. Benchohra and G. M. N'Guerekata, Advanced Fractional Differential and Integral Equations, Mathematics Research Developments, Nova Science Publishers, Inc., New York, 2015.

[4] S. Abbas, M. Benchohra, J. R. Graef and J. Henderson, Implicit Fractional Differential and Integral Equations, De Gruyter Series in Nonlinear Analysis and Applications, 26, De Gruyter, Berlin, 2018.

[5] N. Adjimi And M. BenbachiR, Katugampola fractional differential equation with Erdelyi-Kober integral boundary conditions, Advances in the Theory of Nonlinear Analysis and its Applications, 5(2)(2021), 215228.

[6] R.P. Agarwal, M. Benchohra And S. Hamani, A survey on existence results for boundary value problems of nonlinear fractional differential equations and inclusions, Acta Appl. Math., 109(3)(2010), 973-1033.

[7] R. Almeida, A Caputo fractional derivative of a function with respect to another function, Commun. Nonlinear Sci. Numer. Simul., 44(2017), 460-481.

[8] R. Almeida, Functional differential equations involving the $\psi$-Caputo fractional derivative, Fractal and Fractional, 4(2)(2020), 1-10.

[9] A. Boutiara, M.S. Abdo AND M. Benbachir, Existence results for $\psi$-Caputo fractional neutral functional integro-differential equations with finite delay, Turk. J. Math., 44(2020), 2380-2401.

[10] C. Derbazi, Z. Baitiche, M. Benchohra and A. Cabada, Initial value problem for nonlinear fractional differential equations with $\psi$-Caputo derivative via Monotone iterative technique, axioms 2020, 9, 57.

[11] R. Hilfer, Applications of Fractional Calculus in Physics, World Scientific Publishing Co., Inc., River Edge, NJ, 2000.

[12] A.A. Kilbas, H.M. SRivastava And J.J. Trujillo, Theory and Applications of Fractional Differential Equations, North-Holland Mathematics Studies, 204, Elsevier Science B.V., Amsterdam, 2006.

[13] M.M. MATAR, Existence of solution for fractional Neutral hybrid differential equations with finite delay, Rocky Mountain Journal of Mathematics, 50(6)(2020), 2141-2148.

[14] A.U.K. Niazi, J. Wei, M. Ur Rehman and D. Jun, Existence results for hybrid fractional neutral differential equations, Adv. Difference Equ., 2017, Paper No. 353, 11 pp.

[15] I. Podlubny, Fractional Differential Equations, Mathematics in Science and Engineering, 198, Academic Press, Inc., San Diego, CA, 1999.

[16] F. Si Bachir, S. Abbas, M. Benbachir, Mouffak Benchohra and G.M. N'Guérékata, Existence and attractivity results for $\psi$-Hilfer hybrid fractional differential equations, CUBO, 23(1)(2021), 145-159.

[17] V. E. Tarasov, Fractional Dynamics, Nonlinear Physical Science, Springer, Heidelberg, 2010.

[18] J. Vanterler da C. Sousa and E. Capelas de Oliveira, On the $\psi$-Hilfer fractional derivative, Commun. Nonlinear Sci. Numer. Simul., 60(2018), 72-91. 
Naas Adjimi, Maamar Benbachir and Kaddour Guerbati

[19] Y. Zhou, Basic Theory of Fractional Differential Equations, World Scientific Publishing Co. Pte. Ltd., Hackensack, NJ, 2014.

[20] Y. ZHou, Fractional Evolution Equations and Inclusions: Analysis and Control, Elsevier/Academic Press, London, 2016.

This is an open access article distributed under the Creative Commons Attribution (c) License, which permits unrestricted use, distribution, and reproduction in any medium, provided the original work is properly cited. 